Published in "Journal of Shoulder and Elbow Surgery 27 (4): 635-640, 2018"

which should be cited to refer to this work.

\title{
The posterior ridge of the greater tuberosity of the humerus: a suitable landmark for the posterior approach to the shoulder joint?
}


between the infraspinatus (IS) and teres minor (TM) muscle. ${ }^{14}$ Because the TM runs parallel and adjacent to the IS, it is difficult to identify the plane separating the 2 muscles. ${ }^{1,14}$ Identifying the interval between the IS and TM (IS-TM interval) is crucial, as a dissection carried out more distally to the interval endangers functionally important anatomic structures in the quadrangular and triangular axillary spaces, including the axillary nerve and its muscle branch to the TM, the posterior circumflex humeral artery, and more medially, the suprascapular nerve and circumflex scapular artery. Because of these difficulties, some authors recommend the ISsplitting approach. However, this approach endangers the nerve supply to parts of the IS. $1,8,18,20$

Both the IS and TM belong to the rotator cuff, act as external rotators of the shoulder joint, and are attached to the dorsal aspect of the greater tuberosity. ${ }^{14}$ A closer inspection of the anatomic insertion of the external rotators reveals that there is a prominent ridge posteriorly, just at the distal border of the greater humeral tuberosity, corresponding to a "punctum maximum" where the attachment of the IS merges with the attachment of the TM. With experience in daily surgery and practice during anatomic courses for trainees, this ridge can easily be palpated.

The purposes of this study were (1) to determine the anatomic and topographic relationship between the posterior ridge of the greater tuberosity and the rotator cuff interval that separates the IS and TM (IS-TM interval) and (2) to prove whether - this landmark may be used clinically as a reliable guide during a posterior surgical approach to the glenohumeral joint.

\section{Materials and methods}

This is an anatomic study on 25 formalin-fixed, human cadaveric shoulders; of these, 4 were paired and 17 were unpaired. None had evidence of previous surgery. In 5 cadaveric shoulders (20\%), a full-thickness rotator cuff tear (cuff arthropathy, 4 female shoulders and 1 male shoulder) was present. Of the specimens, 10 were taken from female cadavers (1 paired) and 15 from male cadavers (3 paired). All 25 shoulders were dissected in a prone position. The skin, subcutaneous tissue, and deltoid muscle were removed. The interval between the IS and TM muscle (IS-TM interval), the inferior border of the glenoid (IBG), the posterior ridge (punctum maximum) of the greater tuberosity, and the insertion of the IS and $\mathrm{TM}$ at the greater tuberosity were identified. A line at the level of the IBG parallel to the horizontal line through the acromion and perpendicular to the adducted humeral shaft was drawn (line through the IBG). Structures evaluated and distances measured during dissection included the (1) distance from the posterior ridge of the greater tuberosity to the IS-TM interval, (2) distance from the ridge to the horizontal line through the IBG, and (3) distance from the IBG to the IS-TM interval. Measurements were taken in humeral neutral rotation with a fully adducted arm (Fig. 1).

\section{Statistics}

All statistical analyses were performed with $\mathrm{R}(\mathrm{R}$ : A language and environment for statistical computing; R Foundation for Statistical

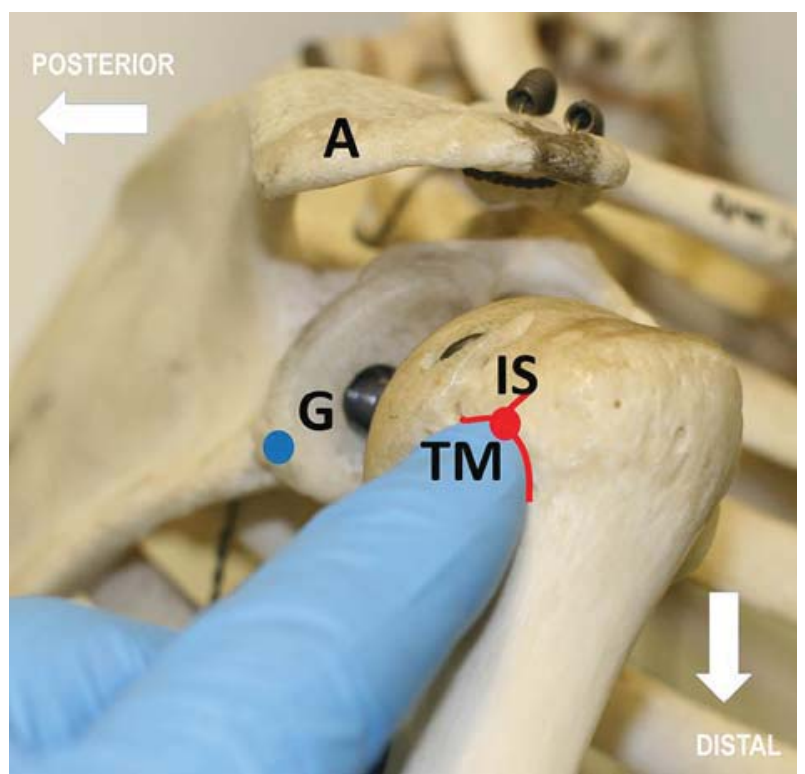

Figure 1 Skeleton model: dorsolateral view of posterior aspect of a right shoulder joint. The posterior ridge of the greater tuberosity (red dot) indicates the border between the infraspinatus (IS) and teres minor muscle $(T M)$. The blue dot indicates the inferior border of the glenoid $(G)$. A, acromion. (For interpretation of the references to color in this figure legend, the reader is referred to the web version of this article.)

Computing, Vienna, Austria; http://www.R-project.org/). Descriptive statistics included means, standard deviations, and ranges. The subgroups were compared by the Mann-Whitney $U$ test. The significance level was set to $\alpha=.05$.

\section{Results}

In all specimens, the posterior ridge of the greater tuberosity, a prominent point on the posterior greater tuberosity lateral to the articular margin (of about half the size of the tip of the index finger), could be identified underneath the deltoid muscle by blunt dissection and palpation (Figs. 1-4). Internal and external rotation of the shoulder joint facilitated the localization of the prominence. The IS-TM interval was further identified after removal of the deltoid muscle. With an approach from lateral, starting at the ridge, the TM muscle separated easily from the IS muscle (Fig. 2). The superficial upper border of the TM muscle belly overlapped the inferior border of the IS. The entry point into the IS-TM interval was always slightly more proximal than the exit in the deep aspect at the level of the glenohumeral joint. Therefore, the IS-TM interval had a vertically oblique direction. In $92 \%$ of specimens (23 of 25 ) for the IS and in $72 \%$ of the specimens (18 of 25) for the TM, a fatty, tendinous raphe was present in the middle section of the muscles (Figs. 2 and 3). These raphes did not entirely separate the IS and TM muscles.

The ridge was located, on average, $3 \mathrm{~mm}$ (range, -2 to $8 \mathrm{~mm}$; SD, $2.4 \mathrm{~mm}$ ) distal to the IS-TM interval for all specimens, $5 \mathrm{~mm}$ (range, $3-8 \mathrm{~mm}$; SD, $1.3 \mathrm{~mm}$ ) distal to the 


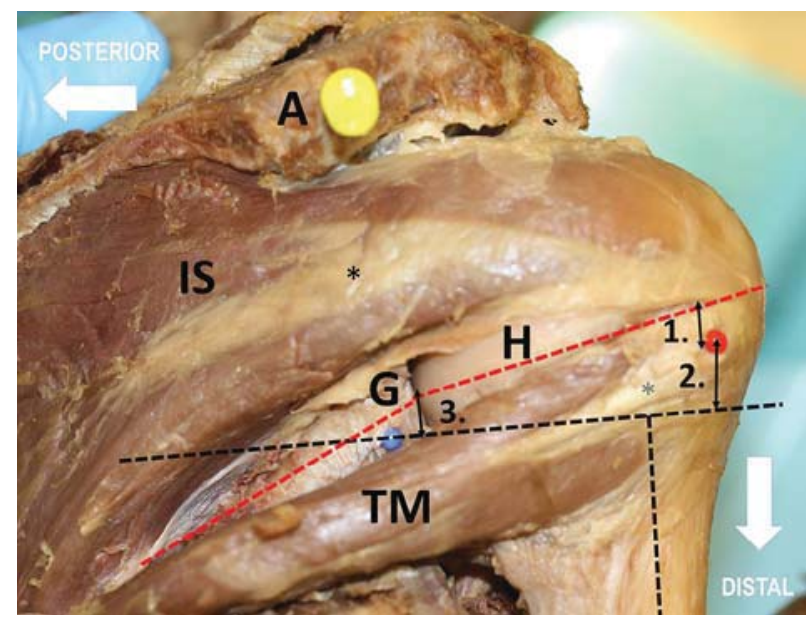

Figure 2 Dorsal view of posterior aspect of a right shoulder. The deltoid muscle has been removed. The black asterisk indicates the raphe of the infraspinatus muscle (IS); gray asterisk, the raphe of the teres minor muscle (TM). $A$, acromion; $G$, glenoid; $H$, humeral head; 1 , distance between posterior ridge of greater tuberosity (red $d o t$ ) and IS-TM interval (dashed red line); 2, distance between posterior ridge of greater tuberosity and horizontal line through inferior border of glenoid (blue dot); 3, distance between IS-TM interval and inferior border of glenoid. (For interpretation of the references to color in this figure legend, the reader is referred to the web version of this article.)

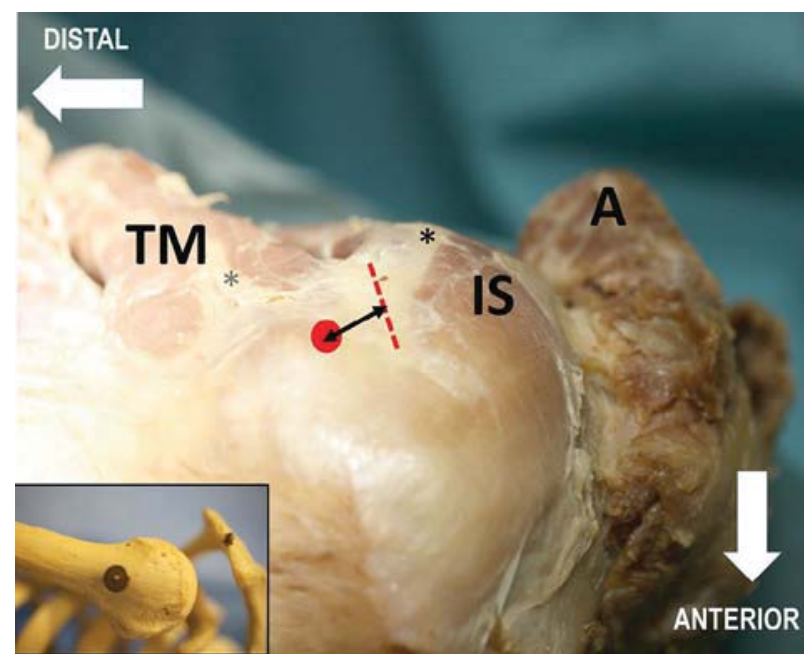

Figure 3 Dorsolateral view of a posterior right shoulder joint (the corresponding skeleton model [inset] is included for better orientation). The deltoid muscle has been removed. The black arrow indicates the distance between the posterior ridge of the greater tuberosity (red dot) and the infraspinatus muscle (IS)-teres minor muscle (TM) interval (dashed red line). The black asterisk indicates the raphe of the IS; gray asterisk, the raphe of the TM. A, acromion. (For interpretation of the references to color in this figure legend, the reader is referred to the web version of this article.)

IS-TM interval in men, and $1 \mathrm{~mm}$ (range, -2 to $5 \mathrm{~mm}$; SD, $2.0 \mathrm{~mm}$ ) distal to the IS-TM interval in women (Table I). The IS-TM interval corresponded to a point $5 \mathrm{~mm}$ (range, $0-10 \mathrm{~mm}$; $\mathrm{SD}, 3.3 \mathrm{~mm}$ ) proximal to the IBG. In all shoulders the ridge

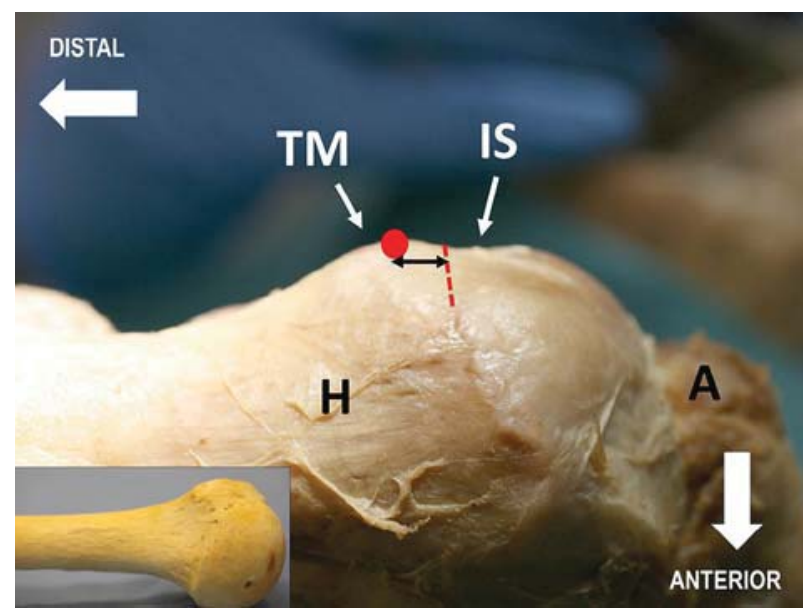

Figure 4 Lateral view of a right shoulder joint (the corresponding skeleton model [inset] is included for better orientation). The deltoid muscle has been removed. The black arrow indicates the distance between the posterior ridge of the greater tuberosity (red dot) and the infraspinatus muscle $(I S)$-teres minor muscle $(T M)$ interval (red dashed line). $H$, humerus; $A$, acromion. (For interpretation of the references to color in this figure legend, the reader is referred to the web version of this article.)

was located a mean of $8 \mathrm{~mm}$ (range, 4-15 mm; SD, $3.1 \mathrm{~mm}$ ) proximal to the IBG (Fig. 2). In specimens with rotator cuff arthropathy (Fig. 5), the distance from the IS-TM interval to the IBG and the distance from the ridge to the IBG were significantly longer, with mean values of $8 \mathrm{~mm}$ (range, 6-10 mm; $\mathrm{SD}, 1.5 \mathrm{~mm}$ ) and $14 \mathrm{~mm}$ (range, 12-15 mm; SD, $1.3 \mathrm{~mm}$ ), respectively. The distance from the ridge to the IS-TM interval did not differ significantly between specimens with rotator cuff arthropathy (mean, $2 \mathrm{~mm}$; range, -2 to $8 \mathrm{~mm}$; $\mathrm{SD}, 3.8 \mathrm{~mm}$ ) and those without rotator cuff arthropathy (mean, $4 \mathrm{~mm}$; range, 0-6 mm; SD, $1.9 \mathrm{~mm}$ ) (Table I).

\section{Discussion}

The posterior approach to the shoulder joint is rarely required; however, it is used for repairs in cases of recurrent dislocation or subluxation of the shoulder, ${ }^{1,9,16}$ glenoid osteotomy, ${ }^{12,15}$ and the treatment of scapular neck and glenoid fractures, as well as for tumor resection. ${ }^{3,7,14}$ Because these surgical procedures are performed infrequently, many surgeons may not have experience with this approach. Detailed anatomy knowledge is imperative to avoid surgical complications. Many different surgical approaches to the posterior shoulder joint have been used. ${ }^{8}$ However, few studies have included detailed descriptions of clear anatomic landmarks relative to the IS-TM interval.

Identifying the IS-TM interval is important as it corresponds to an internervous plane between the IS (suprascapular nerve) and TM (axillary nerve). A dissection proximal to the IS-TM interval may lead to denervation of parts of the IS, ${ }^{8,18}$ and a dissection carried out too far distally endangers important anatomic structures in the quadrilateral or 


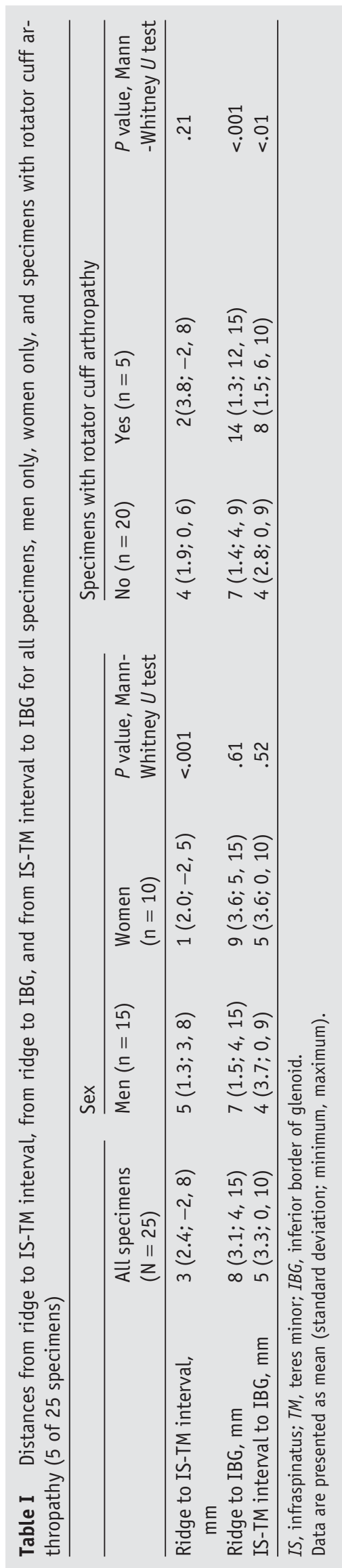

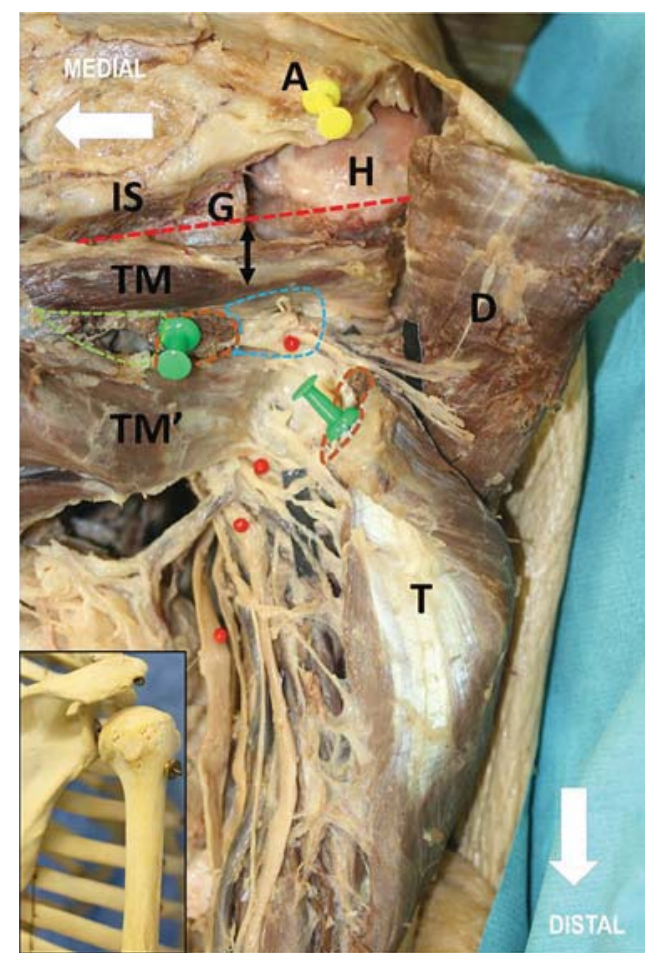

Figure 5 Dorsal view of a dissected right shoulder specimen with cuff tear arthropathy (the corresponding skeleton model [inset] is included for better orientation). The infraspinatus muscle (IS)teres minor muscle (TM) interval (red dashed line) shifts proximally together with the posterior ridge of the greater tuberosity, increasing the distance to the inferior border of the glenoid (black arrow) (Fig. 2). The ridge is hidden by the deltoid muscle $(D)$. The IS shows fatty degeneration. The TM serves as a barrier to important neurovascular structures in the quadrilateral and triangular spaces. The dissected long head of the triceps muscle $(T)$ ( 2 green pins) has been reflected laterally, freeing the view to the axillary and radial nerve (red dots). The quadrilateral and triangular spaces correspond to the blue and green marked areas distal to the TM, respectively. A, acromion (yellow pin); $G$, glenoid; $H$, humerus; $T M^{\prime}$, teres major muscle. (For interpretation of the references to color in this figure legend, the reader is referred to the web version of this article.)

triangular space. ${ }^{2,14}$ To avoid injury to these structures and because the safe IS-TM interval is often difficult to find intraoperatively, ${ }^{1}$ some authors prefer the IS-splitting approach. ${ }^{1,8,18,20}$ Shaffer et a ${ }^{18}$ described the location of a raphe in the IS (Fig. 2) and stated that it could be used to expose the posterior capsule. They found it in all 20 cadaveric shoulders that they examined at an average of $15.4 \mathrm{~mm}$ from the scapular spine and at the level of the midglenoid. The use of this IS-splitting interval provides excellent exposure of the entire posterior glenoid rim with minimal retraction of the IS muscle belly. ${ }^{1}$ Shaffer et al described 2-3 nerve branches to each portion of the IS. In 5 shoulders, there was 1 single large branch supplying parts of the IS. Bailie et $\mathrm{al}^{1}$ also noted that the main branch to the superior part of the IS was located along the base of the scapular spine and crossed the IS raphe at a minimum of $24 \mathrm{~mm}$ medial to the glenoid rim before 
penetrating the muscle. However, the closest branch to the glenoid entered the muscle at an average of $20 \mathrm{~mm}$ from the glenoid rim and always entered at the inferior muscle portion, below the IS raphe. Therefore, splitting the IS may denervate the inferior parts of the IS muscle (Fig. 2). With the use of the IS-splitting approach, any dissection medial to the glenoid must be performed with care to avoid injury to the branches of the suprascapular nerve. The approach cannot be extended. During the dissections in our study, we inconsistently found tendinous raphes both in the IS (92\%) and-less pronounced-in the TM (72\%) (Figs. 2 and 3). Care must be taken not to mix up both structures when an IS-splitting approach is preferred. A dorsal approach to the shoulder through the mistaken TM raphe would be too far distal (Fig. 2).

Previous studies have described the anatomy of the axillary nerve in relation to the posterior approach to the shoulder joint. ${ }^{1,2,4,14}$ Burkhead et $\mathrm{al}^{4}$ measured the distance from the axillary nerve to a point $1 \mathrm{~cm}$ medial to the posterolateral corner of the acromion in 10 fresh cadaveric shoulders and found it to be, on average, $55 \mathrm{~mm}$ with the arm in a neutral position; with abduction, this distance decreased significantly. The distance was highly dependent on sex (shorter in specimens taken from female cadavers). Bailie et $\mathrm{al}^{1}$ performed a cadaveric study on 14 paired cadaveric shoulders and found that the average distance from the posterolateral corner of the acromion to the axillary nerve was $65 \mathrm{~mm}$ in a neutral position. However, the distance was as close as $53 \mathrm{~mm}$ in a small specimen taken from a woman. In all shoulders, this distance decreased significantly in humeral extension and abduction. Because of these variations, the posterolateral corner of the acromion might not be suitable to use clinically as a guide during surgery.

Our study revealed that a prominent ridge at the posterior tuberosity, just at the distal border of the greater humeral tuberosity, can be consistently identified. Similar to the lesser tuberosity for the subscapular muscle, the posterior tuberosity for the IS and TM increases the lever arm for rotation. The ridge begins at a point $1-5 \mathrm{~mm}$ below the point where the attachment of the IS merges with the attachment of the TM. The portion of the greater tuberosity that abuts the anatomic neck shows 3 flattened impressions, also called "facets." $5,6,10,11,13,17,19$ Thereby, the middle and the lowest facets serve the IS and TM muscles as an attachment site. The posterior ridge of the greater tuberosity has been mentioned neither as a prominent bony landmark nor as a suitable guide to identify the rotator cuff interval between the IS and TM. With the aid of the posterior ridge of the greater tuberosity, the ISTM interval can reliably be located. The ridge can easily be palpated through a small deltoid-splitting incision and further located by blunt dissection and passive rotation of the humeral head. Therefore, the ridge defines a reproducible landmark that can help in accessing the posterior shoulder safely in the internervous plane between the IS and TM. In contrast to the IS-splitting approach, the medial enlargement of the IS-TM interval does not put the nerve supply to the IS at risk (Fig. 2). In contrast to other landmarks, the ridge is less dependent on sex, body size, and position of the glenohumeral joint as the ridge - and also the IS-TM interval-moves with the position and migration of the humeral head. There is a sex difference in the distance from the ridge to the IS-TM interval of only $4 \mathrm{~mm}$. In other words, in both female and male patients, the IS-TM interval can be expected just $1 \mathrm{~mm}$ (female) to $5 \mathrm{~mm}$ (male) above the ridge (5 $\mathrm{mm}$ [range, 3-8 $\mathrm{mm}$; SD, $1.3 \mathrm{~mm}$ ] in male patients and $1 \mathrm{~mm}$ [range, -2 to $5 \mathrm{~mm}$; SD, $2 \mathrm{~mm}$ ] in female patients). Moreover, the ridge is even able to act as a suitable guide in patients with rotator cuff arthropathy. As with these pathologies, if the humeral head shifts proximally (Fig. 5), the ridge will do the same. This explains why in contrast to healthy specimens, in specimens with cuff tears, the distances from the ridge to the IBG and from the IS-TM interval to the IBG increased with cranial migration of the humeral head (Table I). By starting the dissection of the IS-TM interval at the posterior ridge of the greater tuberosity in a horizontal line, separation of the IS from the TM can be carried out at a safe distance from the suprascapular nerve, which runs $15-25 \mathrm{~mm}$ medial to the glenoid rim. Therefore, the surgeon must be aware of the vertically oblique direction of the internervous plane between the IS and TM muscle bellies from proximal to distal.

\section{Conclusion}

The anatomic data presented in this study reveal that the posterior ridge of the greater tuberosity (part of the greater humeral tuberosity) serves as a suitable landmark to find the internervous plane between the IS and TM. This ridge should not be crossed distally unnecessarily. In contrast to previously described landmarks, the posterior ridge of the greater tuberosity moves with the position and migration of the humeral head. It is less dependent on the patient's sex, size, and arm position and the quality of the rotator cuff. During dissection using the posterior approach to the shoulder joint in the IS-TM interval, if the starting point is at the ridge, the risk to the suprascapular nerve is minimized. This approach can be safely enlarged medially. It gives access to the posterior shoulder joint always proximal to the IBG. The posterior ridge of the greater tuberosity further indicates a prominence proximal to the insertion of the TM and a point always proximal to the IBG. An approach carried out from this landmark will always be a safe distance from important anatomic structures in the quadrilateral or triangular space.

\section{Disclaimer}

The authors, their immediate families, and any research foundations with which they are affiliated have not received any financial payments or other benefits from any commercial entity related to the subject of this article. 


\section{References}

1. Bailie DS, Moseley B, Lowe WR. Surgical anatomy of the posterior shoulder: effects of arm position and anterior-inferior capsular shift. J Shoulder Elbow Surg 1999;8:307-13.

2. Ball CM, Steger T, Galatz LM, Yamaguchi K. The posterior branch of the axillary nerve: an anatomic study. J Bone Joint Surg Am 2003;85:1497-501.

3. Bartonícek J, Tucek M, Lunácek L. Judet posterior approach to the scapula. Acta Chir Orthop Traumatol Cech 2008;75:429-35.

4. Burkhead WZ, Scheinberg RR, Box G. Surgical anatomy of the axillary nerve. J Shoulder Elbow Surg 1992;1:31-6.

5. Charpy A, Nicolas A. Musles de l'épaule. In: Traité d'anatomie humaine. Paris: Masson et Cie; 1912. p. 385-8.

6. Davies DV, Coupland RE. Osteology. In: Gray's anatomy. 34th ed. Harlow, UK: Longmans, Green, and Co; 1967. p. 400-4.

7. Ebraheim NA, Mekhail AO, Padanilum TG, Yeasting RA. Anatomic considerations for a modified posterior approach to the scapula. Clin Orthop Relat Res 1997;(334):136-43.

8. Fucentese SF, Jost B. Posteriorer Zugang zum Schultergelenk [Posterior approach to the shoulder]. Oper Orthop Traumtol 2010;22:188-95 [in German]. http://dx.doi.org/10.1007/s00064-010-8064-3

9. Fuchs B, Jost B, Gerber C. Posterior-inferior capsular shift for the treatment of recurrent, voluntary posterior subluxation of the shoulder. J Bone Joint Surg Am 2000;82:16-25.

10. Gegenbaur C. Muskeln und Gliedmassen. In: Lehrbuch der anatomie des menschen. Leipzig: Verlag von Wilhelm Engelmann; 1899. p. 267-9, 414, 415.

11. Hayek H. Muskeln des Oberarms. In: Anatomischer Atlas für Studierende und Ärzte. 1st ed. Vienna: Urban \& Schwarzenberg; 1963. p. 316.
12. Hernandez A, Drez D. Operative treatment of posterior shoulder dislocations by posterior glenoidplasty, capsulorrhaphy, and infraspinatus advancement. Am J Sports Med 1986;14:187-91.

13. Hollinshead WH. Pectoral region, axilla, shoulder, and arm. In: Textbook of anatomy. 3rd ed. New York: Harper and Row; 1974. p. 171-5, 203-4.

14. Hoppenfeld S, deBoer P, Buckley R. Surgical exposures in orthopaedics. The anatomic approach. Philadelphia: Lippincott Williams \& Wilkins; 2009.

15. Ortmaier R, Moroder P, Hirzinger C, Resch H. Posterior open wedge osteotomy of the scapula neck for the treatment of advanced shoulder osteoarthritis with posterior head migration in young patients. J Shoulder Elbow Surg 2017;26:1278-86. http://dx.doi.org/10.1016/j.jse.2016 .11 .005

16. Pogorzelski J, Braun S, Imhoff AB, Beitzel K. Open-wedgeglenoidosteotomie als therapie bei posteriorer schulterinstabilität aufgrund vermehrter glenoidretroversion [Open-wedge osteotomy of the glenoid for treatment of posterior shoulder instability with increased glenoid retroversion]. Oper Orthop Traumatol 2016;28:438-48 [in German]. http://dx.doi.org/10.1007/s00064-016-0457-5

17. Rouvière H II. Squelette du bras. Humerus. In: Anatomie humaine, descriptive et topographique. 2nd ed. Paris: Libraires de l'Académie de Médecine; 1924. p. 10-3.

18. Shaffer BS, Conway J, Jobe FW, Kvitne RS, Tibone JE. Infraspinatus muscle-splitting incision in posterior shoulder surgery. An anatomic and electromyographic study. Am J Sports Med 1994;22:113-20.

19. Spalteholz W. Knochen der oberen Gliedmassen. In: Handatlas der Anatomie des Menschen. 5th ed. Leipzig, Germany: Verlag von S. Hirzel; 1907. p. 95-7.

20. Wirth MA, Butters KP, Rockwood CA. The posterior deltoid-splitting approach to the shoulder. Clin Orthop Relat Res 1993;(296):92-8. 